\title{
Study on Route Selection System of Heavy-Cargo Highway Transportation based on ArcGIS Engine
}

\author{
Yanei Zhou ${ }^{1, a}$, Chuanhua Zeng ${ }^{1, b}$, Fei Xian ${ }^{1, c}$ \\ ${ }^{1}$ School of Automobile and transportation, Xihua University, Chengdu 610039,China \\ a915455549@qq.com, bzchfirst@263.net, c345782263@qq.com
}

Keywords: ArcGIS Engine; route selection system;network analysis technology

\begin{abstract}
In this paper, the route selection system of heavy-cargo highway transportation was the secondary developed based on ArcGIS Engine. The spatial data platform was set up by simulating the actual road network, and combined with quantitative analysis of the influence factors, a route planning model of double objective was established. Finally, the optimal route was determined by using ArcGIS network analysis technology.
\end{abstract}

\section{Introduction}

The selection of transport routes is the major work in the early period of heavy-cargo highway transport. The optimal transportation lines can be quickly and scientifically determined by using the computer technology, which will greatly shorten the construction period and cost savings.As a computer system,GIS is used for collection,simulation, processing,query retrieval,analysis and expression of geographic data,and it has strong capability of spatial information processing and spatial data analysis ${ }^{[1]}$. In this paper, the route selection system of heavy-cargo highway transportation is redeveloped based on ArcGIS and Visual studio 2010. The spatial data platform was set up by simulating the actual road network, then the transportation time, cost and safety factors of evaluation index can be written into the property field based on the arc-node topological data structure, and a certain weight of road network can be gotten.Combined with quantitative analysis of the influence factors, the multi-objective optimization model based on route is established, and the multi-objective optimal path and graphical display are gotten by adopting the platform of network analysis module ${ }^{[2]}$.

\section{Establish the model of route selection of heavy-cargo highway transport}

Influence factors of route selection of heavy-cargo highway transport. According to the special requirements of heavy-cargo highway transport, the main factors of affecting the transport route selection are clearance restrictions of route, road pavement and administrative restrictions.Clearance restrictions mainly refers to the space restrictions in height and width, Road pavement restrictions mainly refer to the minimum curve radius and ramp,road pavement and bridges ldams loading capacity that required by vehicles and equipment in the transport route. Administrative restrictions mainly refer to the constraints of heavy-cargo transportation vehicles and equipment during transporting by relevant transport regulations of local transport authorities. When the actual route selection was conducted, in addition to the above factors, economically feasibility, the shortest time as well as the shortest transportation distance and other factors should also be taken into consideration, so that the transport task can be completed economically, efficiently and safely.

Building model. When making heavy-cargo highway transportation, there are usually a variety of transport routes between origin and destination.Supposed that there are $\mathrm{N}$ cities from origin to destination, and every two adjacent cities are viewed as a road section, then a road network can be 
obtained.Supposed that there are $\mathrm{j}(\mathrm{j}=1,2,3, \ldots, \mathrm{m})$ feasible routes from the origin to destination, each possible route contains $\mathrm{i}(\mathrm{i}=1,2,3, \ldots, \mathrm{n}) \mathrm{road}$ sections, dual objective linear model is established by taking the time and cost as objective function.

$$
\begin{aligned}
& T_{j}=\sum_{i=1}^{n}\left(t_{r i}+t_{c i}+t_{h i}+t_{w i}+t_{l i}+t_{i}\right)+t_{j} \quad(\mathrm{i}=1,2,3 \ldots, \mathrm{n} ; \mathrm{j}=1,2,3, \mathrm{~m}) \\
& \left\{\begin{array}{l}
t_{r i}=t_{r m i}+t_{r j i}+t_{r c i} \\
t_{c i}=t_{c m i}+t_{c j i} \\
t_{h i}=t_{h m i}+t_{h j i}+t_{h c i} \\
t_{w i}=t_{w m i}+t_{w j i}+t_{w c i} \\
t_{l i}=t_{l s i}+t_{l v i}+t_{l r i} \\
t_{i}=l_{i} / \bar{v}_{i}
\end{array}\right.
\end{aligned}
$$

In which, ${ }^{T}{ }_{j}$ is the total time of complete transport tasks on route $\mathrm{j} ; t_{r i},{ }_{c i},{ }^{t_{h i}},{ }^{t_{w i}}$ and ${ }^{t_{l i}}$ is the time of eliminating the limit of road pavement,radius,height,width and bridges ldams loading capacity; $t_{i}$ is the transportation time on the road section $\mathrm{i}$; ${ }_{j}$ is time of complete other transport tasks on route $\mathrm{j} ;{ }^{l}{ }_{i}$ is the length of road section $\mathrm{i}(\mathrm{km}) ; \bar{v}_{i}$ is the average speed $(\mathrm{km} / \mathrm{h})$ of transporting on the road section $i$.

$$
\begin{aligned}
& F_{j}=\sum_{i=1}^{n}\left(f_{r i}+f_{c i}+f_{h i}+f_{w i}+f_{l i}+f_{J i}+f_{i}\right)+f_{j} \\
& \left\{\begin{array}{l}
f_{r i}=k_{r m} \cdot l_{r m i}+k_{r j} \cdot l_{r j i}+k_{r c} \cdot l_{r c i} \\
f_{c i}=k_{c m} \cdot x_{c m i}+k_{c j} \cdot x_{c j i} \\
f_{h i}=k_{h m} \cdot x_{h m i}+k_{h j} \cdot l_{h j i}+k_{h c} \cdot l_{h c i} \\
f_{w i}=k_{w m} \cdot x_{w m i}+k_{w j} \cdot l_{w j i}+k_{w c} \cdot l_{w c i} \\
f_{l i}=k_{l s} \cdot l_{l s i}+k_{l v} \cdot l_{l v i}+k_{l r} \cdot l_{l r i} \\
f_{J i}=k_{J i} \cdot x_{J_{i}} \\
f_{i}=l_{i} \cdot \overline{f_{i}}
\end{array}\right.
\end{aligned}
$$

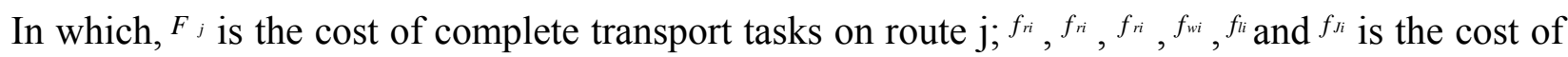
eliminating the limit of road pavement,radius,height ,width,bridges \dams loading capacity and the administration requirements. $f_{l}$ is the vehicle fuel cost of transporting on the road section $\mathrm{i}_{\text {; }} f_{j}$ is cost of complete other transport tasks on route $\mathrm{j} ; \overline{f_{/}}$is the average vehicle fuel cost $(\mathrm{RMB} / \mathrm{km})$ of transporting on the road section $i$.

\section{Design the system}

System requirements analysis. The route selection system of heavy-cargo highway transport based on ArcGIS Engine basically has the following requirements: (1)Clearly know the information of bulk cargo, including the size, weight, shape, material, etc;(2) Accurate clearly grasp the road network, including roads and end point, road grade, line mileage, pavement type and load, bend radius, the number of bridge and tunnel, etc; (3) Graphical display of feasible routes; (4) The feasible scheme to adjust.

System function design. As an applied information system, in addition to the basic file operations function, the route selection system of heavy-cargo highway transportation also should have the editing capability of cargo and highway information, the formation of feasible routes and optimal 
route and graphical display. The system function structure is shown in figure 1 .

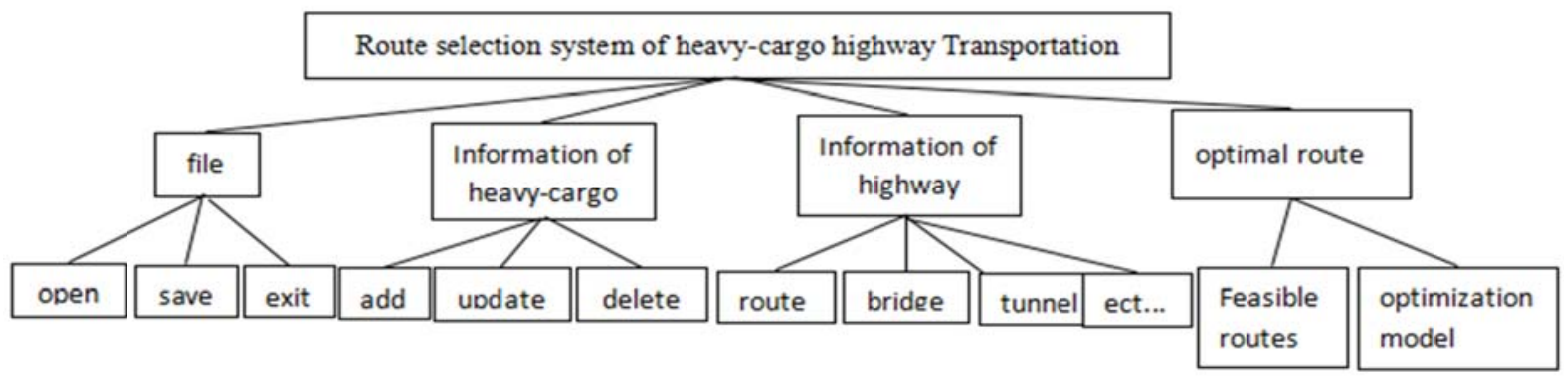

Fig.1 System function structure

System database design. The data source of route selection system of heavy-cargo highway transportation stores in two databases, one is the spatial database, and the other is the attribute database. The two databases are linked up through corresponding fields, realizing seamless connection of spatial information and attribute information about the large highway transportation route choice $^{[3]}$. Spatial database is mainly graphic data of map, and stores map layer and basic information such as graphics, as shown in figure 2. The attribute data mainly includes the basic information of the description cargo and highway linear features and the data of ancillary facilities, as shown in figure3.

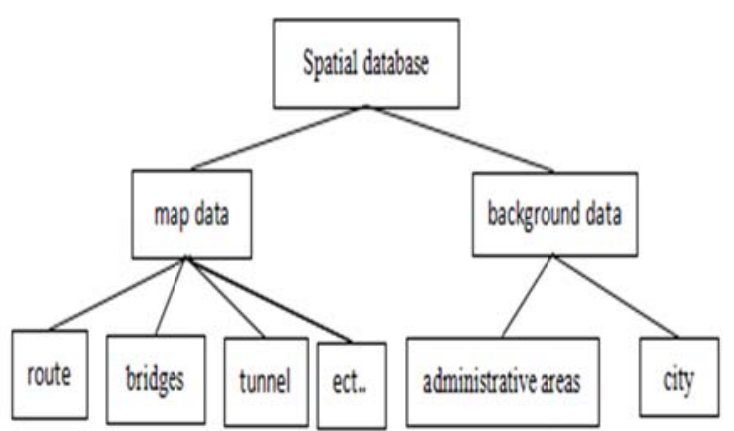

Fig.2 Spatial database

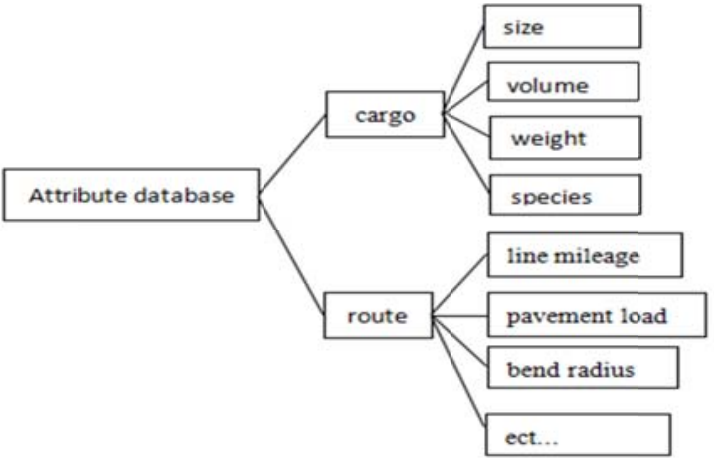

Fig.3 Attribute database

\section{Realization the system}

The system main interface design.According to the requirements analysis and function design of the route selection system of heavy-cargo highway transportation, the main interface design of the system operation is mainly distribution layout of each function module, respectively corresponding to the related development controls of ArcGIS Engine. The "layer control" panel which is on the left of the interface places the TOCControl control which is used to view and modify the layer information; The right side of the interface is the main map window, have two functions such as map and plate making, which is realized through the MapControl control and used to display graphics and basic operation. The system main interface is shown in figure 4 .

Feasible routes generated interface design.According to ArcGIS to simulate actual road network structures, and the space road network is set up. Then combined with time-cost double objective optimization model, and adopting the platform network analysis module to calculate, the least comprehensive costs and the shortest travel possible route are gotten. As shown in figure 5. 


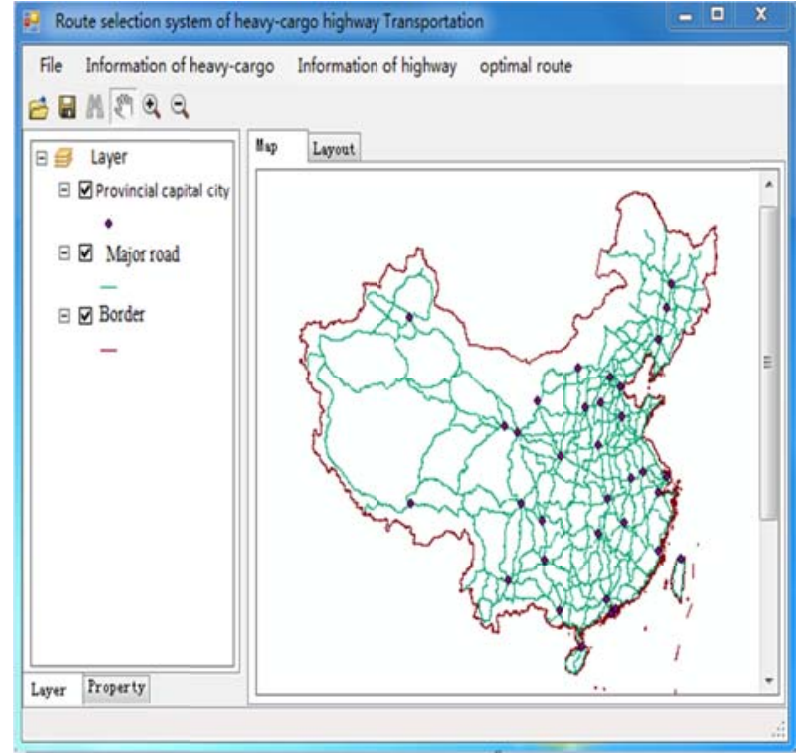

Fig.4 Main interface

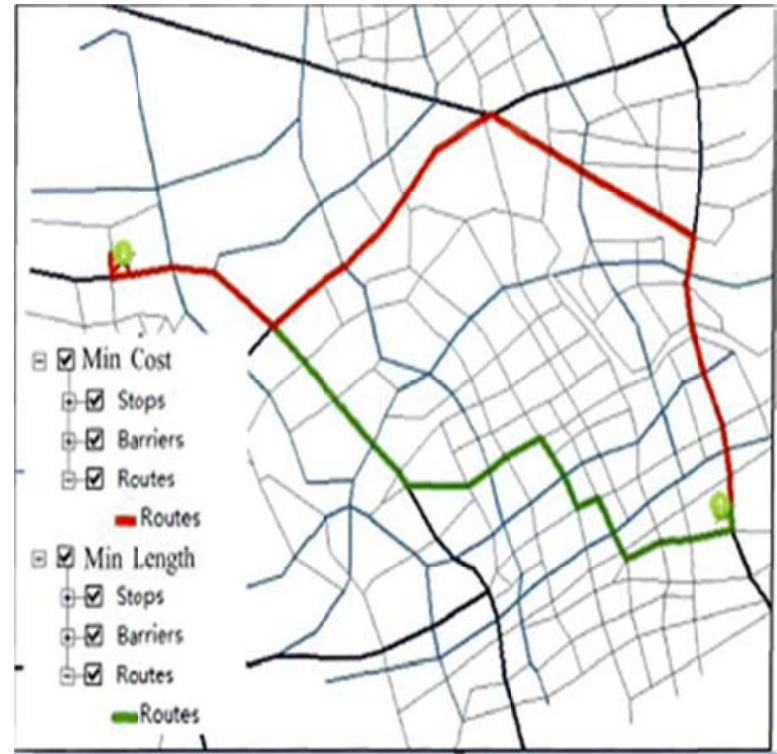

Fig.5 Feasible routes generated interface

\section{Summary}

Based on heavy-cargo highway transportation as the background, this paper studies the problem of how to choose reasonably transportation lines. The optimum transportation route selection system is established by simulating transportation road network based on ArcGIS Engine and Visual Studio2010, which provides a solution to choose the reasonable transportation line for decision makers at the beginning of the transportation.

\section{Acknowledgement}

In this paper, the research was sponsored by the Graduate student innovation fund of Xihua University(Project No. Ycjj2015151 and Ycjj2015038).

\section{References}

[1]Fen Li.Study on Some Problems of Highway Alignment intelligent Optimization based on GIS[D].Chang,an University,2008.

[2]Chunge Yang.Study on the Route Selection about Heavy-Cargo Highway Transportation based on ArcGIS[D].Southwest Jiaotong University,2014.

[3]Ping Yang.Study on Large-scale Equipment Transportation Route Selection of Highway[D].Xihua University,2013. 